\title{
Modélisation du déroulement d'activités humaines en mer côtière. Scénarios appliqués à la mer d'Iroise
}

\author{
Matthieu Le TiXerant*, Françoise Gourmelon*, Gerard Veron** \\ *Laboratoire Géomer (UMR LETG 6554 CNRS), IUEM, Technopôle Brest-Iroise, 29280 Plouzané \\ **Laboratoire de Biologie Halieutique, Ifremer, Technopôle Brest-Iroise, 29280 Plouzané
}

\begin{abstract}
RESUME
Les mers côtières, franges marines des zones côtières, sont particulièrement concernées par les activités humaines qui interagissent entre elles et avec leur environnement. Afin d'analyser ces interactions, le laboratoire Géomer (LETG UMR 6554 CNRS) a développé une plate-forme de modélisation de la Dynamique des Activités Humaines (DAHU) qui comporte un module adapté au déroulement d'activités humaines en milieu marin. Cet article présente les applications développées en mer d'Iroise. Elles sont fondées sur l'élaboration de scénarios plausibles visant à fournir des éléments relatifs aux activités humaines (1) dans leur déroulement quotidien, (2) en cas d'événement accidentel (3) ou dans le cas d'aménagement d'infrastructures.
\end{abstract}

MOTS CLES : Mer côtière, activités humaines, modélisation spatio-temporelle, scénarios, aide à la gestion

\begin{abstract}
Coastal seas, marine part of the coastal zone, are particulary attractive for human society. Human activities involve interactions that can lead to conflicts and to ressources degradation. In order to analyse the coastal system, the Geomer laboratory has developed a modelling platform of Human Activities Dynamic (DAHU) which contains a specific module adapted to the human marine activities (DAHU-MAM). This paper presents applications developed on the Iroise Sea (France). Three scenarios are proposed in an operational coastal management objective : (1) highlighting potential conflicts of use, (2) impact of a shipwrek (3) or impact of a substructure installation.
\end{abstract}

KEY WORDS : Coastal sea, human activities, spatio-temporal modeling, scenarios, coastal management

\section{Introduction}

Les mers côtières, franges marines des zones côtières, apparaîssent actuellement comme un milieu en voie de socialisation rapide sous l'effet de la multiplication des usages (Corlay 2001; Trouillet 2004). En effet, elles subissent de multiples pressions liées aux nombreuses activités humaines qui s'y déroulent (pêches professionnelles, transport maritime, extraction de matériaux, exercices militaires, rejets de dragage, aménagement d'infrastructures (récifs artificiels, éoliennes offshore...), activités de loisir...). Ces différentes formes d'occupation de l'espace et d'exploitation du milieu créent un jeu complexe d'interactions pouvant conduire à des conflits entre usagers et à la dégradation de l'environnement. L'analyse de ces interactions est actuellement un des objectifs majeurs des recherches menées sur la zone côtière dans la perspective de sa gestion intégrée (Cuq 2000; Dronkers et al. 1999). Dans ce contexte, l'étude des modes d'utilisation et d'exploitation du milieu par l'Homme dans l'espace et dans le temps s'impose (Bartlett 1999).

La plate-forme de simulation de la Dynamique des Activités Humaines (DAHU) (Cuq et al. 2002; Tissot 2003) a pour objectif de décrire la distribution spatio-temporelle d'agents représentatifs d'activités humaines en zone côtière. Un module adapté à la spécificité du déroulement d'activités humaines en milieu marin, DAHU-MAM (Module Activités Marines), a été développé dans un objectif opérationnel d'aide à la gestion de la mer côtière (Le Tixerant 2004). De manière à tester l'approche méthodologique, cette contribution présente différents scénarios développés sur la mer d'Iroise. Ils ont pour objectif de simuler des situations plausibles et d'évaluer leurs conséquences sur le déroulement des activités de la zone d'étude. 


\section{Méthodologie}

La démarche proposée par la plate-forme DAHU est fondée sur le fait que le déroulement des activités humaines est conditionné par de multiples contraintes de nature environnementale, météorologique, juridique, sociale et économique (Le Tixerant et al. 2006). DAHU identifie chaque contrainte de pratique par un filtre spatio-temporel (figure 1). La prise en compte simultanée de l'ensemble des filtres conduit à une modélisation spatio-temporelle du déroulement des activités permettant, pour chaque activité, d'aboutir à :

- $\quad$ un Territoire de Pratique Potentielle (TPP) qui identifie les zones où l'activité est susceptible de se pratiquer en fonction des contraintes qui conditionnent spatialement son déroulement ;

- un Calendrier de Pratique Potentielle (CPP) qui identifie des périodes au cours desquelles l'activité est susceptible de se pratiquer en fonction des contraintes qui conditionnent temporellement son déroulement ;

- des données statistiques qui quantifient le déroulement de l'activité.

La phase de simulation consiste à intégrer les informations préalablement mises en forme dans une Base d'Information Géographique pilotée par un SIG. Dans sa conception, le simulateur est doté d'une architecture s'inspirant directement d'un Système Multi-Agents (SMA). En effet, chaque activité est représentée par un agent autonome doté d'un comportement archétypique qui varie en fonction des conditions de pratique lors de la période de simulation sélectionnée. Le système est interfacé avec le SIG dont il dépend pour la mise en forme des données d'initialisation et pour l'exploitation des résultats en sortie. Ainsi, suite à une phase de simulation, les résultats obtenus se présentent sous la forme de cartes qui synthétisent les informations relatives au déroulement quotidien des activités.

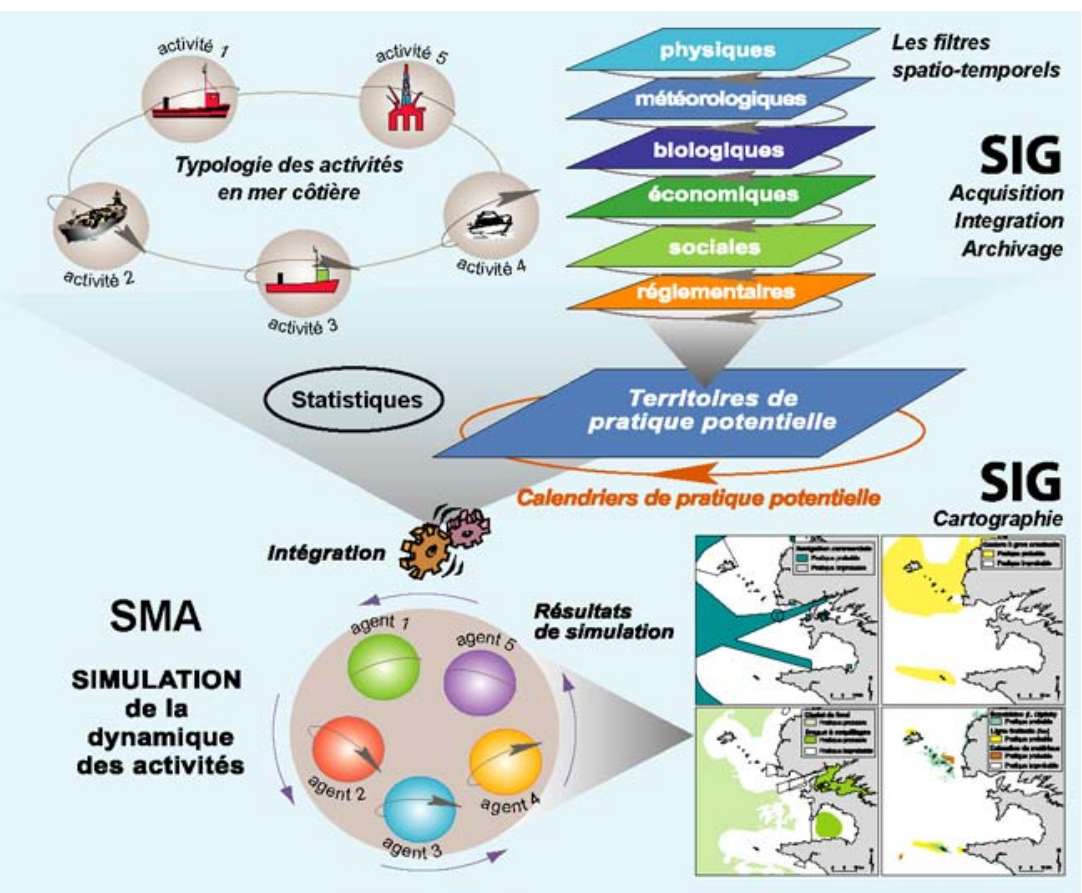

Figure 1. Principes méthodologiques

DAHU-MAM permet l'élaboration de scénarios plausibles qui peuvent notamment concerner :

- le déroulement simultané de plusieurs activités marines ;

- les conséquences d'une impossibilité temporaire d'accès occasionnée par un évènement (accident en mer, intervention sur épaves fuyantes, exercices militaires...) intervenant à un instant donné et provoquant des perturbations sur le déroulement des activités habituellement présentes sur la zone ;

- l'impact d'un aménagement d'infrastructures (éoliennes, plateforme, récifs artificiels...) entraînant des modifications durables du déroulement des activités de la zone.

\section{Applications en mer d'Iroise}

L'approche méthodologique a été testée en mer d'Iroise, qui constitue un cas d'étude pertinent du fait des multiples activités potentiellement conflictuelles qui s'y déroulent (Mission-PNMI 2005).

Suite à la réalisation d'une typologie des activités en mer d'Iroise et à un inventaire des informations existantes, nous avons choisi de travailler sur les principales activités de pêche professionnelle, de navigation maritime et d'extraction de matériaux. Les sources d'information varient selon les activités. Concernant la pêche professionnelle, activité prépondérante en mer d'Iroise, une collaboration a été mise en place avec le Laboratoire de Biologie Halieutique d'Ifremer afin de bénéficier des données générées par le Système d'Informations Halieutiques (Berthou et al. 2002) et des enquêtes réalisées auprès des pêcheurs de la zone (Boncoeur et al. 2002). Concernant la navigation maritime, les informations ont été recueillies principalement auprès du CROSS-Corsen et des principaux sémaphores bordant la mer d'Iroise. Les données relatives aux extractions de matériaux proviennent essentiellement de la réglementation en vigueur.

L'information géographique de référence (trait de côte, bathymétrie, faciès biosédimentaire, réglementation) est issue de la Base d'Information Géographique "Iroise" développée au laboratoire Géomer (Le Berre 1999). 


\subsection{Scénario "déroulement d'activités"}

DAHU-MAM permet la mise en évidence du déroulement d'activités au cours de l'année en fonction des conditions de pratique. Cette simulation peut s'appliquer à plusieurs activités ayant des déroulements distincts dans le temps et dans l'espace. Elle met en évidence des interactions positives (activités complémentaires) ou négatives (à l'origine de conflits d'usage potentiels).

A l'ouest de la mer d'Iroise, les fonds marins, constitués de graviers et de sables, sont propices au chalutage. Cet espace offre également des potentialités aux fileyeurs qui doivent toutefois tenir compte des courants assez forts (notamment en période de vives eaux). Ce résultat de simulation (figure 2) montre que les activités du filet et du chalut peuvent se dérouler au même moment sur des zones identiques, d'où des conflits d'usage potentiels. Pourtant les incidents recensés sont peu fréquents car des accords de cohabitation informels ont été établis par les pêcheurs. En fonction des coefficients de marée, des zones sont réservées soit aux chalutiers soit aux fileyeurs.

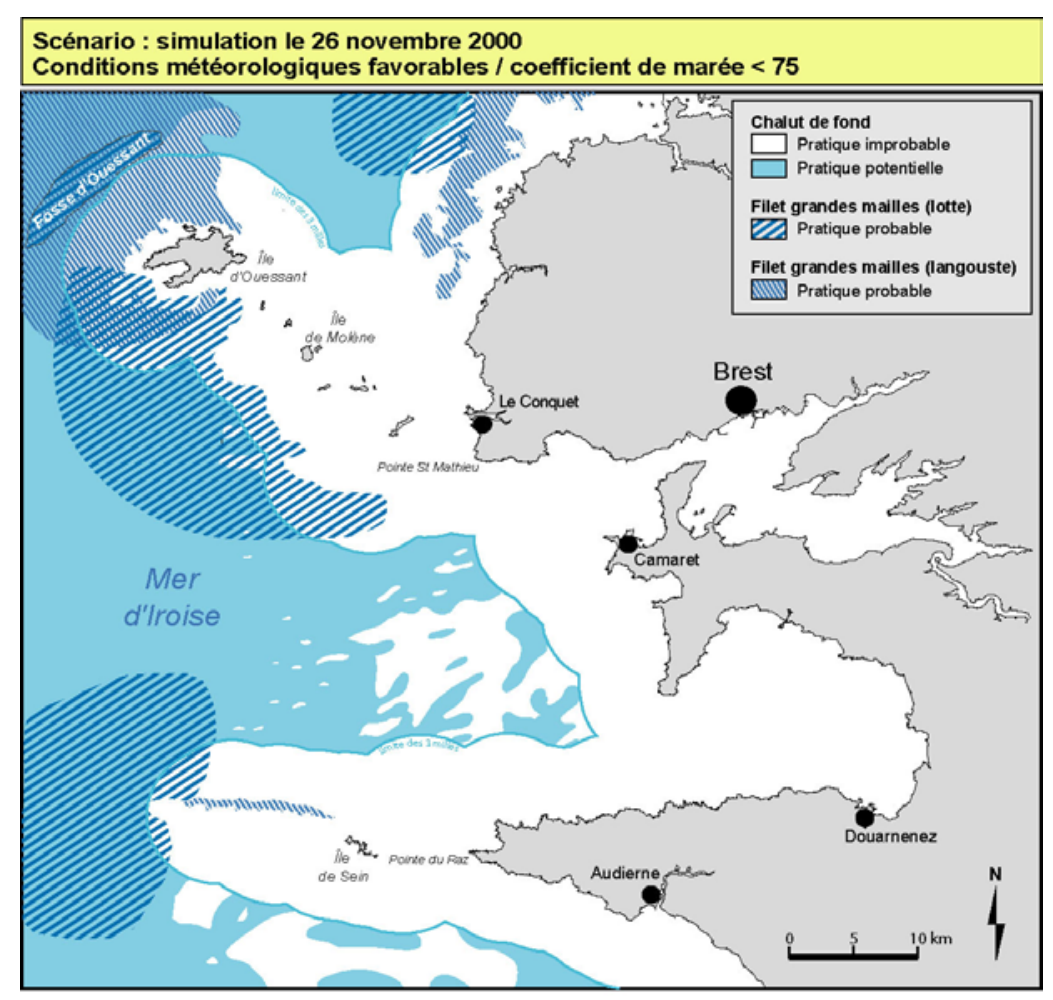

Figure 2. Simulation du chalut de fond et du filet grandes mailles

\subsection{Scénario "naufrage"}

Lors d'une pollution accidentelle, les autorités compétentes peuvent prendre la décision d'interdire pendant une période déterminée, la pratique de la pêche sur une zone donnée ${ }^{1}$. Il peut alors être utile d'évaluer l'impact potentiel sur le déroulement des activités et l'incidence économique que pourrait engendrer cette mesure temporaire :

- en temps réel, pour les autorités qui doivent prendre la décision d'interdiction de pratique de la pêche (durée de l'interdiction, aide à la définition des zones de protection prioritaire) ;

- $\quad$ et ultérieurement, lors de la mise en place d'un éventuel processus d'indemnisation des pêcheurs.

Les résultats de simulation produits par DAHU-MAM permettent d'accéder à ce type d'information en mettant en évidence les activités directement concernées par la mesure, le nombre de jours de pêche perdus ainsi que le nombre de navires concernés (figure 3 ).

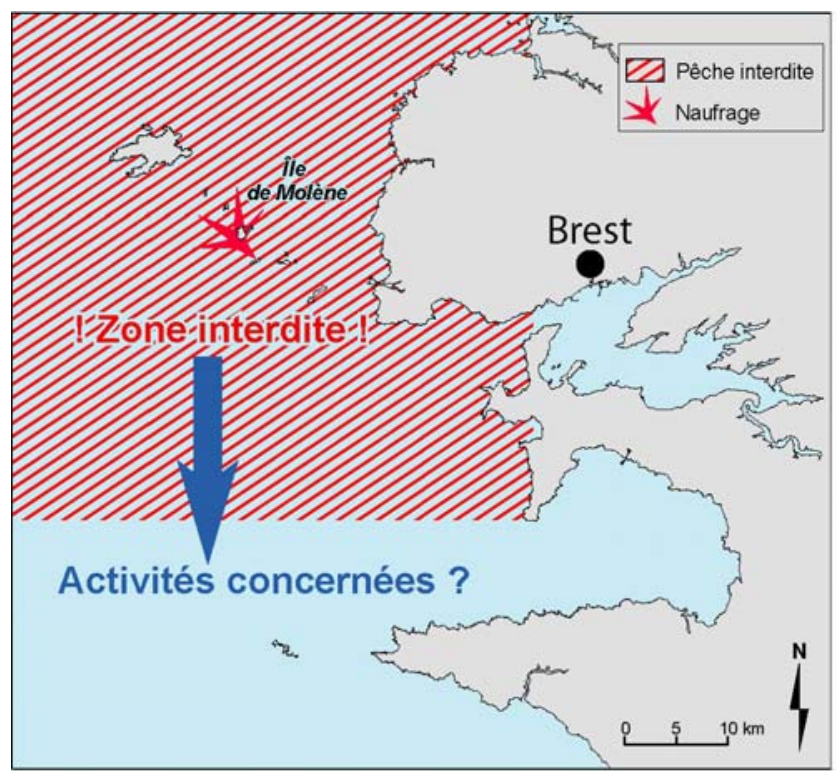

\begin{tabular}{|l|c|c|}
\hline Métiers concernés & $\begin{array}{c}\text { Jours de } \\
\text { pêche perdus }\end{array}$ & $\begin{array}{c}\text { Nombre de } \\
\text { navires concernés }\end{array}$ \\
\hline Chalut de fond & 30 & 43 \\
Chalut pélagique & 30 & $<10$ \\
Drague à coquillages & 30 & 60 \\
Filet grandes mailles & 20 & 74 \\
Filet petites mailles & 20 & 97 \\
$\begin{array}{l}\text { Casier à grands } \\
\text { crustacés }\end{array}$ & 20 & 63 \\
\hline
\end{tabular}

Figure 3. Simulation d'un naufrage avec déversement de matière dangereuse le 5/10/2000 entrainant la fermeture d'une zone à la pêche pendant 30 jours

\footnotetext{
${ }^{1}$ Instruction POLMAR du 4 mars 2002
} 


\subsection{Scénario "éoliennes en mer"}

Les engagements de la France en matière d'émissions de gaz à effet de serre ${ }^{2}$ imposent une politique volontariste de développement des énergies renouvelables. Disposant du second potentiel européen de production d'électricité à partir de centrales éoliennes en mer, cette filière est donc susceptible de se développer (ADEME-CLAROM 2002).

Dans le cas d'un projet d'implantation d'éoliennes en mer, les études d'impact abordent les contraintes techniques (mesure de vent, puissance de l'installation, plan de maintenance, raccordement au réseau), environnementales (impacts de l'installation sur le milieu) et socio-économiques (Kannen et al. 2004). Sur ce dernier point, il est notamment nécessaire d'évaluer l'impact potentiel des installations sur les activités humaines présentes qui peuvent être classées en deux groupes selon le degré de perturbation (CA-OWEE 2001).

- Les activités susceptibles d'être très affectées par l'implantation d'éoliennes en mer sont la navigation maritime et la pêche pratiquée à l'aide d'arts traînants. Du fait des risques de collisions, les principales voies de navigation et les chenaux d'accès aux ports sont évidemment à proscrire. La pratique des arts traînants (chaluts, dragues, lignes...) est également susceptible d'être fortement dérangée par l'implantation d'éoliennes en mer. Il peut en être de même pour des activités exploitant des espaces très restreints où l'implantation d'éoliennes pourrait quasiment rendre impossible le déroulement des activités.

- Les activités susceptibles d'être moins affectées par l'implantation d'éoliennes en mer sont la pêche pratiquée à l'aide d'arts dormants (filets, casiers) ${ }^{3}$ et la pêche de loisir.

Pour mettre en évidence une « zone optimale d'installation » d'un projet de parc éolien en mer d'Iroise, les territoires de pratique potentielle des activités humaines présentes sont superposés en fonction du degré de perturbation engendré. La simulation conduit à l'évaluation des risques de conflits potentiels suivant les zones d'implantation ${ }^{4}$ (figure 4).
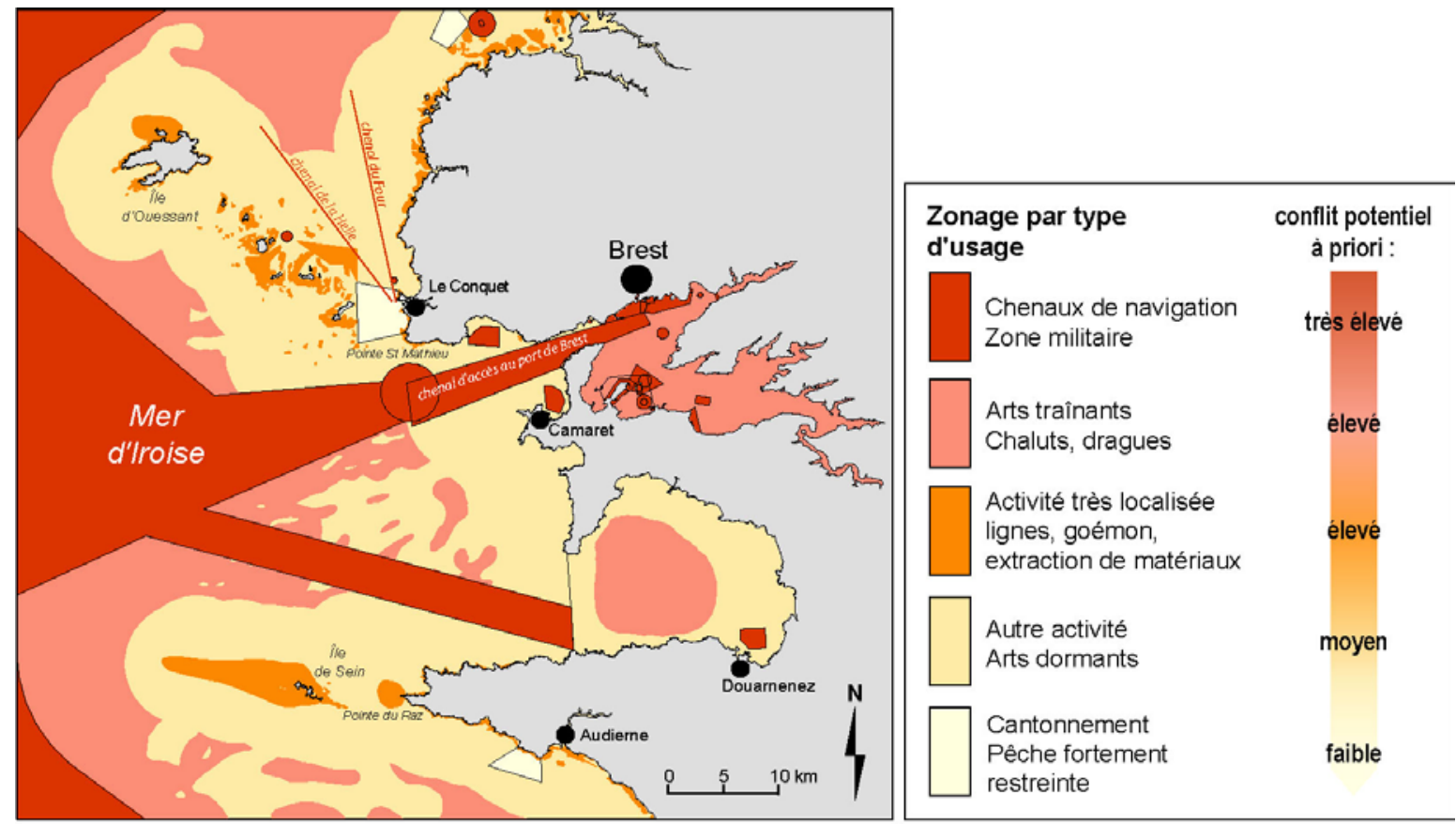

Figure 4. Zone optimale d'installation pour un parc éolien en mer d'Iroise du point de vue des risques de conflits d'usage

Les résultats de simulation montrent que le contexte multi-usage de la mer d'Iroise est une des contraintes majeures que devrait prendre en compte un projet d'implantation d'éoliennes sur cette zone. Néanmoins elle n'est pas la seule car les caractéristiques physiques (la bathymétrie notamment) ne sont pas très propices à ce type d'installation. En effet, pour éviter des coûts de construction et de maintenance trop élevés, les éoliennes devraient être implantées à proximité de la côte pour bénéficier des faibles profondeurs (de préférence inférieures à 30 mètres) sachant que les secteurs abrités tels que la rade de Brest et dans une moindre mesure la baie de Douarnenez possèdent des potentiels éoliens faibles du fait de la turbulence des vents. De plus, pour chaque site d'implantation, des contraintes environnementales, paysagères, techniques et économiques devraient bien sûr être intégrées au projet.

\footnotetext{
2 Directive européenne du 27 septembre 2001 relative à la promotion de l'électricité produite à partir de sources d'énergies renouvelables qui prévoit pour la France un objectif indicatif de consommation d'électricité produite à partir d'énergies renouvelables de $21 \%$ à l'horizon 2010, contre $15 \%$ en 1997.

${ }^{3}$ Les fondations des éoliennes peuvent être conçues pour devenir des récifs artificiels et donc avoir éventuellement des effets bénéfiques sur la ressource

${ }^{4}$ Nous avons admis, pour ce scénario, que les contraintes techniques et financières n'étaient pas limitantes.
} 


\section{Conclusion}

Dans un objectif opérationnel, il est indispensable que les gestionnaires soient à même de comprendre l'organisation et le déroulement des activités humaines sur leur territoire de compétence, ce qui implique une approche pluridisciplinaire et innovante fondée sur la modélisation. Ce type de démarche permet notamment l'élaboration de scénarios susceptibles d'alimenter les prises de décision des acteurs de l'action publique :

- en illustrant la variabilité du déroulement des activités au cours de l'année et notamment en mettant en évidence des zones de conflits potentiels ;

- $\quad$ en apportant une aide à la décision suite à un évènement exceptionnel tel qu'un naufrage, ou dans le cas d'un projet pouvant modifier le déroulement des activités présentes sur la zone tel qu'un projet d'implantation d'éoliennes.

Suite à la validation des informations produites, un des objectifs actuels du projet est de transférer aux gestionnaires la méthodologie et la technologie développées dans le cadre de DAHU-MAM afin de les assister dans la production, la mise en cohérence et la communication d'informations pertinentes et indispensables à une gestion efficace des activités humaines en mer côtière. La démarche pourrait permettre de mettre en relief l'interdépendance entre les différents éléments et acteurs du système et s'avérer pertinente dans le cadre d'une gestion concertée de la zone côtière (Pennanguer 2005), en offrant aux acteurs locaux des éléments pour promouvoir des concertations sur le terrain qui devront être relayées par les outils institutionnels de gestion.

\section{Bibliographie}

ADEME-CLAROM, 2002. Document de cadrage. Séminaire ADEME-CLAROM / Etat des lieux du développement de l'éolien offshore, Rueil-Malmaison.

Bartlett D., 1999. Working on the frontier of Science : applying GIS to the Coastal Zone, Marine and Coastal GIS, Wright \& Bartlett, Taylor \& Francis, p. 11-24.

Berthou P., Daurès F., Guyader O., Merrien C., Leblond E., Demaneche S., Jézéquel M., Guégan F., Bermell S., Van Iseghem V., 2002. Synthèse des pêcheries 2000. Flottes de Mer du Nord, Manche, Atlantique. Système d'informations halieutiques., Rapport Ifremer (Brest)

Boncoeur J., Alban F., Appéré G., Bermell S., Berthou P., Curtil O., Demanèche S., Drogou M., Guyader O., Huet J., Jézéquel M., Sabourin A., Thébaud O., Véron G., 2002. Activités halieutiques et activités récréatives dans le cadre d'un espace à protéger : le cas du Parc National de la Mer d'Iroise, CEDEM/IFREMER, Brest.

CA-OWEE, 2001. Offshore Wind Energy. Ready to power a sustainable europe. Duwind 2001.006, Delft University of Technology, The Netherlands, Delft University Wind Energy Research Institute (Duwind).

Corlay J.P., 2001. Interactions fonctionnelles et spatiales en zone côtière : réflexions pour l'analyse et la gestion. Cinquièmes Rencontres halieutiques de Rennes. ENSAR/Ifremer, Rennes, p. 69-86.

Cuq F., 2000. Systèmes d'information géographique et gestion intégrée des zones côtières. CoastGIS'99. Ifremer/SHOM, Brest, p. $18-30$.

Cuq F., Bourcier P., 2002. Contribution méthodologique pour le couplage de modèles qualitatifs et quantitatifs au sein d'un Système d'Information Géographique, Rapport final PEVS (CNRS).

Dronkers J., Vries I., 1999. Integrated coastal management: the challenge of transdisciplinarity. Journal of Coastal Conservation, volume 5, p. 97-102.

Kannen A., Gee K., Glaeser B., 2004. Offshore Wind Farms, spatial planning and the German ICZM strategy. Littoral 2004, Aberdeen (Scotland), p. 450-455.

Le Berre I., 1999. Mise au point de méthodes d'analyse et de représentation des interactions complexes en milieu littoral. Thèse de géographie, Université de Bretagne Occidentale, Brest, 236 p.

Le Tixerant M., 2004. Dynamique des activités humaines en mer côtière ; application à la mer d'Iroise. Thèse de géographie, Université de Bretagne Occidentale, 210 p.

Le Tixerant M., Gourmelon F., 2006. Approche dynamique du déroulement d'activités humaines en mer côtière. Cybergeo : Revue européenne de géographie, p. 16.

Mission-PNMI, 2005. "Richesses de l'Iroise" / Activités humaines en Iroise. Préfecture maritime de l'Atlantique / Préfecture du Finistère, $78 \mathrm{p}$.

Pennanguer S., 2005. Incertitude et concertation dans la gestion de la zone côtière. Thèse de doctorat halieutique, Agrocampus Rennes (Département halieutique) ; Université de Bretagne Occidentale (CEDEM) ; Portances-Conseil (SARL), 374 p.

Tissot C., 2003. Evaluation de la variabilité des activités humaines dans l'espace et dans le temps. Application à l'étude des pratiques agricoles intensives dans le département du Finistère. Thèse de géographie, Université de Bretagne Occidentale, Brest, $234 \mathrm{p}$.

Trouillet B., 2004. La "mer côtière" d'Iroise à Finisterre. Etude géographique d'ensembles territoriaux en construction. Thèse de doctorat de géographie, Université de Nantes, 293 p. 